\title{
Publishing in the digital age: Challenges and requirements for authors and users of SpringerLink
}

\author{
Gertraud Griepke \\ Vice President SpringerLink Operations, Springer-Verlag, GmbH, Tiergartenstr. 17, 69121 Heidelberg, \\ Germany \\ Tel.: +496221487 8457; Fax: +4962214878364 \\ E-mail: gertraud.griepke@springer.com \\ URL: www.springerlink.com
}

\section{Introduction}

The use of technology to support the digital production, distribution and storage of scientific content in full-text formats poses challenges for authors and publishers as well as for the librarians and scientists who access the materials. While scientific publishers still have to produce high-quality print products, they have had to develop new production systems that are now able to produce book and journal pages in both print and digital form.

This paper will discuss how Springer organizes its digital production and distribution systems and some of the challenges we will face in the future.

\section{From the manuscript to the final paper - tagging}

All of the world's scientific publishers have agreed on a standard set of XML tags, called metadata, to identify all of the various data elements - journal title, ISSN or ISBN for books, volume and issue number etc. - that can be used in scientific books and journals. Authors can insert some of these tags as they compose their works, but the publishers insert most of the tags.

Graphics are also identified and linked with the appropriate part of the work. Standards for tagging and handling supplementary materials like videos, chemical structures and animation are still in the discussion stage among publishers and other interested parties within the publishing community.

\subsection{HTML or PDF format?}

The other issue that is being debated by the scientific community for journal articles is how the content will be displayed in electronic form. Most publishers and search engine vendors prefer HTML but user surveys indicate that the scientists who will read the articles in electronic form or as printouts prefer 
the PDF format by wide margins. There are strong arguments for both, and it is too early to tell which format will be the final choice.

\section{From the manuscript to the final paper - publication}

A Digital Object Identifier (DOI) is added to each article after copyediting and tagging. The DOI is a persistent electronic identifier that can connect the paper to other publications through cross-reference links. More than 25 million DOIs have been assigned since the program started in 2000.

The actual typesetting process is electronic, and the online version of the paper is published first. Corrections after publication are always in the form of ERRATA, as content cannot be removed from the database of digital articles.

\section{The universe of online publishing}

Springer's electronic content is distributed through a data delivery system to its own website, springerlink.com, and parts of the content are distributed to approximately 100 different parties, which include bibliographic services and some large library consortia that store content on their own sites.

Springer supports and promotes its online content through springer.com, an extensive website that includes catalogs, promotional materials, and information for booksellers and librarians. There are tools for journal authors to communicate with journal editors and other helpful functions for customers to access.

\subsection{SpringerLink}

All of Springer's content that is in electronic form is available online at springerlink.com. Individuals and institutions can subscribe. A subscription includes a comprehensive search capability and, of course, extensive links to electronic journals in other publishers' databases.

Libraries have additional requirements that include individual usage statistics within their institutions. Springer provides these statistics in the COUNTER format, which libraries use to compare usage across multiple publishers.

Finally, SpringerLink is the website that searchers arrive at when they come across a reference to a Springer online journal. These search queries come from a number of different websites, but the Google portal had the highest referral rates of any other portal last year.

\section{Long-term outlook}

In the future we can expect to find increasing amounts of the world's literature being published in electronic form, and sometimes exclusively in electronic form. Searching will be even more intuitive and will be facilitated by semantic links; that is, users will be able to search for similar content. They will also be able to use mathematical formulae to compute graphs and tables, and chemical formulae will be searchable by structure.

In order to make all of this happen, publishers will have to make sure that the content we prepare for publication today will be ready for the enhanced storage, retrieval and searching techniques of the future. 


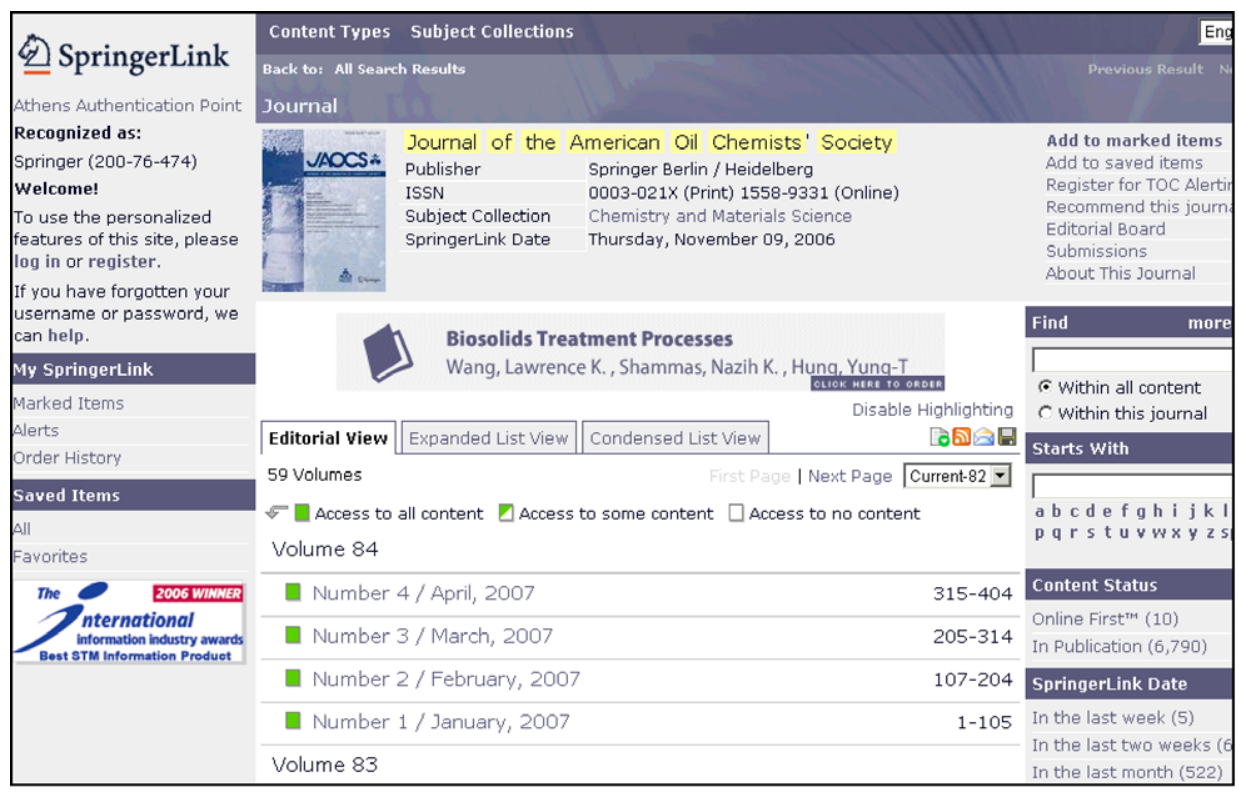

Fig. 1. 